\title{
SOME CONSIDERATIONS ON TUNNELING LOSSES IN FIELD-EFFECT DEVICES FOR LOW-VOLTAGE MICROCONTROLLERS
}

\author{
M. A. GRADO-CAFFARO* and M. GRADO-CAFFARO \\ Scientific Consultants, C/Zulio Palacios, 11, \\ 9B $\mathrm{B}$ 28029-MADRID (SPAIN)
}

(Received 8 November 1997; In final form 23 January 1998)

\begin{abstract}
The loss power density associated with the tunneling current in a typical MOS cell with a floating gate is evaluated for high electric-field strengths in the oxide layer. Furthermore, problems related to oxide thickness are discussed.
\end{abstract}

Keywords: Loss power density; tunneling current; MOS cell; oxide thickness

\section{INTRODUCTION}

The tunneling current in MOS cells for low-voltage microcontrollers based upon EEPROM plays a crucial role in understanding the physical electronics of those field-effect devices. The above current is very sensitive to the thickness of the oxide layer in a given MOS cell because the electric field in this layer depends on the above thickness and the current depends strongly on the electric-field strength [1-4].

On the other hand, it is important to recall that $\mathrm{Al}-\mathrm{SiO}_{2}-\mathrm{Si}$ devices with floating gate will be considered. In effect, these devices are the crucial elements in low-voltage microcontrollers based on EEPROM. These devices have two gates: the control gate and the floating gate [1-4]. When the drain voltage is reduced from $5 \mathrm{~V}$ to $3 \mathrm{~V}$, the corresponding charge pump output voltage also decreases [2, 3]; consequently, the control gate voltage becomes smaller. This fact

*Corresponding author. 
implies a decreasing of the voltage drop across the tunnel oxide [2, 3]. The diminished tunneling of carriers onto the floating gate originates a slow erase operation giving rise to EEPROM fallout [2, 3].

In the following, we will consider $n$-channel devices and we will study the loss power density associated with the tunneling current for relatively high electric-field strengths in the oxide layer. At this point, we can claim that the oxide thickness plays a significant role; in fact, some problems related to this parameter will be discussed very briefly.

\section{THEORETICAL FORMULATION}

The magnitude of the tunneling current density in question obeys the following relationship $[1-5]$ :

$$
J=\frac{e^{3} E^{2} m_{0} E_{g}^{-3 / 2}}{16 \pi^{2} \hbar m_{n}^{*}} \exp \left[-\frac{4\left(2 m_{n}^{*} E_{g}^{3}\right)^{1 / 2}}{3 e \hbar E}\right]
$$

where $e$ is the electron charge, $E$ is the magnitude of the electric-field strength in the oxide layer of the $n$-channel MOS considered, $m_{0}$ is the electron rest-mass, $m_{n}^{*}$ is the tunneling electron effective mass, $\hbar$ is the reduced Planck constant and $E_{g}$ is the barrier height of $\mathrm{Si}$ and $\mathrm{SiO}_{2}$ at cathode side.

If $E$ is sufficiently high, the exponential in Eq. (1) approaches unity and we can conserve the preexponential term so that we can write:

$$
J \approx e^{3} E^{2} E_{g}^{-3 / 2} /\left(16 \pi^{2} \hbar\right)
$$

since $m_{n}^{*} \approx 1.1 m_{0}$ (silicon).

On the other hand, the associated power density $\left(W / \mathrm{m}^{3}\right)$ is given by $P=J E$ so that by virtue of Eq. (2) we have:

$$
P \approx \frac{e^{3} E^{3} E_{g}^{-3 / 2}}{16 \pi^{2} \hbar}
$$

Since $E=V_{\mathrm{ox}} / t_{\mathrm{ox}}$ (where $V_{\mathrm{ox}}$ is the voltage drop across the tunnel oxide and $t_{\mathrm{ox}}$ is the oxide thickness), formula (3) is a good approximation because, in practice, $t_{\mathrm{ox}}$ must be small (typically 
$t_{\mathrm{ox}} \approx 100 \AA$ A; see, for example, Ref. [6]) so that $E$ becomes high. Then, from Eq. (3) it follows:

$$
P \approx \frac{e^{3} V_{\mathrm{ox}}^{3} E_{g}^{-3 / 2}}{16 \pi^{2} \hbar t_{\mathrm{ox}}^{3}}
$$

that is to say, if $t_{\mathrm{ox}}$ is sufficiently small, tunneling losses per unit volume are inversely proportional to $t_{\mathrm{ox}}^{3}$.

On the other hand, by taking into account that $V_{\text {ox }}$ decreases when the drain voltage is reduced from $5 \mathrm{~V}$ to $3 \mathrm{~V}$ (see the introduction of this paper), then $E$ is not too high and, as a consequence, formula (3) is not applicable. Certainly, decreasing of $V_{\text {ox }}$ and a relatively small value of $t_{\mathrm{ox}}$ produce a moderate value of $E$; for a discussion on values of $t_{\mathrm{ox}}$, see Refs. [2,6]. At any rate, optimization of the tunnel oxide thickness constitutes a crucial problem. Variation in critical dimension on this thickness influences the so-called erase coupling ratio and program coupling ratio which determine the voltage induced onto the floating gate from the control gate and the drain, respectively [2]; these ratios are used to evaluate EEPROM cells [2]. In particular, the erase coupling ratio, that is, the fraction of the control gate voltage induced onto the floating gate is [2]:

$$
R_{e}=C_{\mathrm{fc}} / C
$$

since [2]:

$$
\frac{C_{\mathrm{fc}}}{C}=\frac{V_{\mathrm{fg}}}{V_{\mathrm{cg}}}
$$

where $C$ is the total capacitance on the floating gate which is given by:

$$
C=C_{\mathrm{s}}+C_{\mathrm{fc}}+C_{\mathrm{fs}}+C_{\mathrm{d}}
$$

where $C_{\mathrm{s}}$ is the capacitance of the floating gate to the source, $C_{\mathrm{fc}}$ is the capacitance of the floating gate to the control gate, $C_{\mathrm{fs}}$ is the capacitance of the floating gate to the substrate, and $C_{\mathrm{d}}$ is the capacitance of the floating gate to the drain. On the other hand, $V_{\mathrm{fg}}$ is the floating gate voltage and $V_{\mathrm{cg}}$ is the control gate voltage which is induced onto the floating gate during the erase operation. 
With respect to the program coupling ratio, $R_{P}$, we have [2]:

$$
R_{P}=C_{d} / C
$$

Also we have [2]:

$$
\frac{V_{\mathrm{fg}}}{V_{\mathrm{d}}}=\frac{C_{\mathrm{d}}}{C}
$$

where $V_{\mathrm{d}}$ is the drain voltage. From formulae (8) and (9) it follows:

$$
R_{P}=\frac{V_{\mathrm{fg}}}{V_{\mathrm{d}}}
$$

Finally, by virtue of Eq. (4) and by the fact that both $C_{\mathrm{d}}$ and $C_{\mathrm{fc}}$ are inversely proportional to $t_{\mathrm{ox}}$, it follows that $P$ is approximately proportional, on the one hand, to $C_{\mathrm{d}}^{3}$ and, on the other hand, to $C_{\mathrm{fc}}^{3}$ when $t_{\mathrm{ox}}$ is sufficiently small.

\section{CONCLUDING REMARKS}

Formula (4) in conjunction with the considerations associated with the capacitances involved in the above exposition, may be considered as the nucleus of the paper. On the other hand, we wish to remark that our formulation can be used to understand the problems related to the electrical parameters of the $5 \mathrm{~V}$ and $3 \mathrm{~V}$ microcontrollers with EEPROM of Motorola; these problems have been reported in Ref. [2] and their relation to the tunnel oxide thickness constitutes a crucial question.

\section{References}

[1] Chen, I., Holland, S. and Hu, C. (1985). IEEE Trans. Electron Dev., ED-32, 413.

[2] Ku, J. W. and Chao, S. C., Semiconductor Int., May 1997, 105-110.

[3] Grado-Caffaro, M. A. and Grado-Caffaro, M., Act. Pass. Electronic Comp. (in press).

[4] Grado-Caffaro, M. A. and Grado-Caffaro, M., Solid-St. Electronics (submitted).

[5] Sze, S. M., Physics of semiconductors devices (Wiley, 1969).

[6] Ajika, N., Ohi, M., Arima, H., Matsukawa, T. and Tsubouchi, N., IEDM Tech. Dig., 115 (1990). 

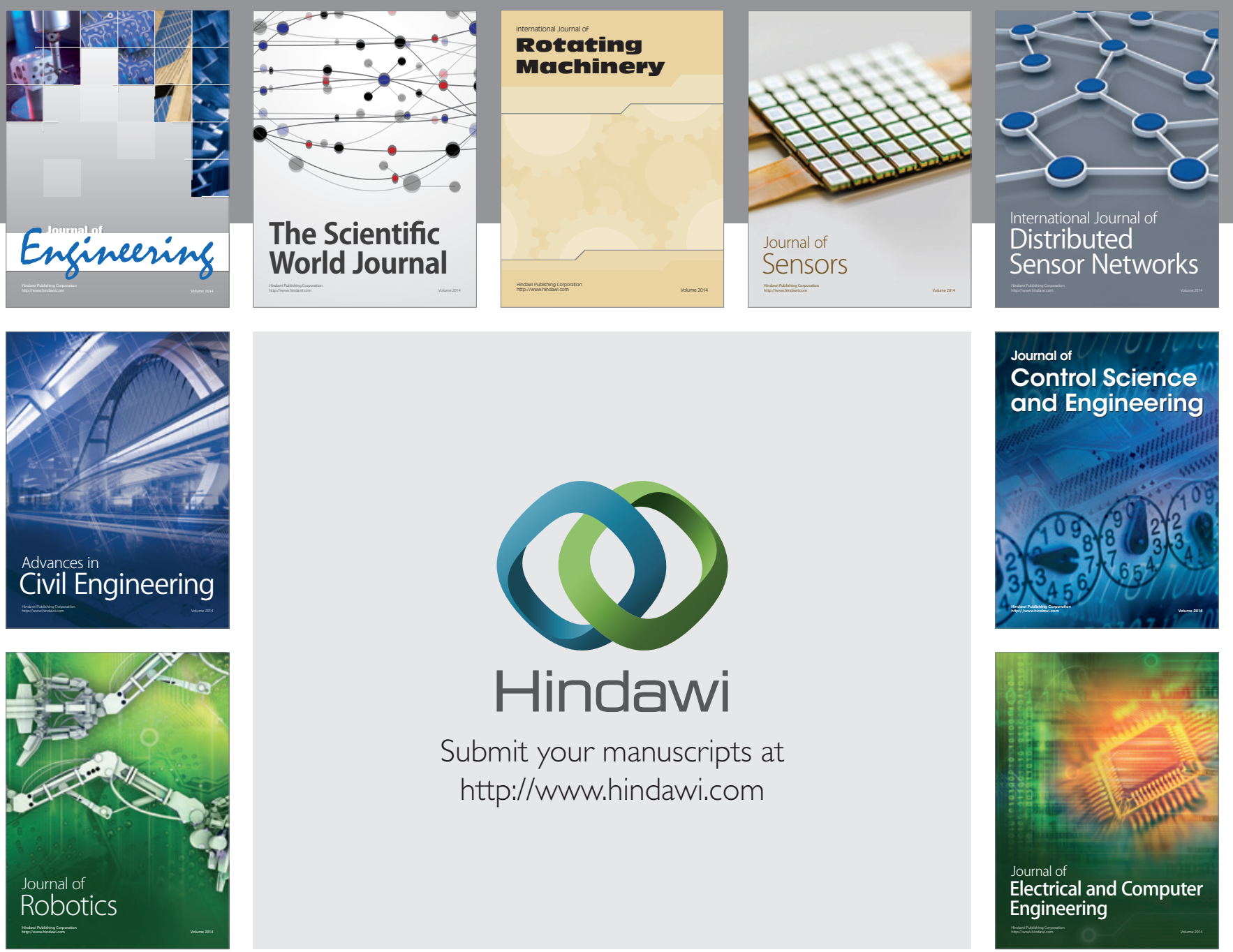

Submit your manuscripts at

http://www.hindawi.com
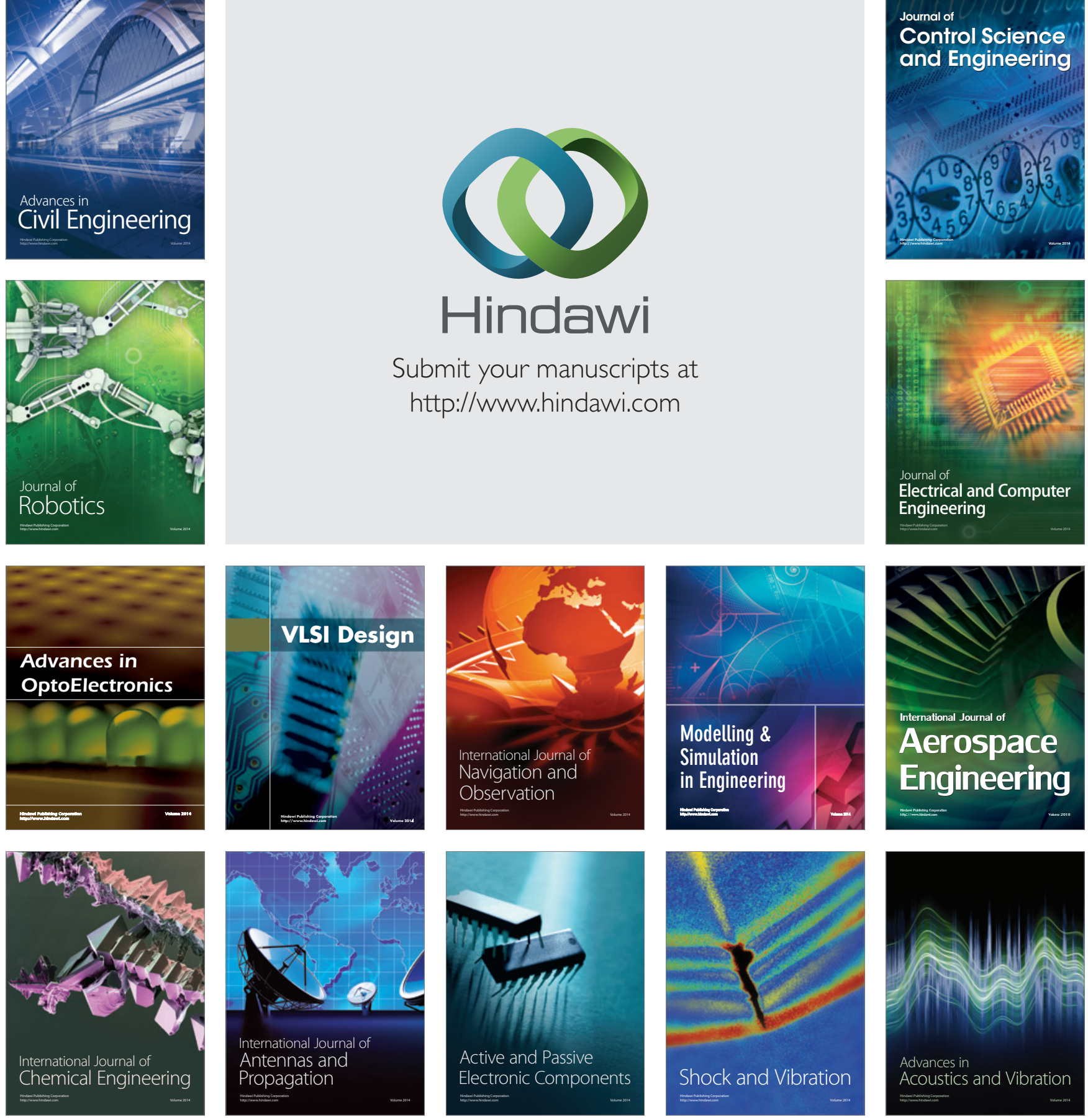